\title{
Use of a formal assessment instrument for evaluation of resident operative skills in pediatric neurosurgery
}

\author{
Caroline Hadley, BS, Sandi K. Lam, MD, MBA, Valentina Briceño, RN, Thomas G. Luerssen, MD, \\ and Andrew Jea, MD \\ Division of Pediatric Neurosurgery, Texas Children's Hospital and Department of Neurosurgery, Baylor College of Medicine, \\ Houston, Texas
}

OBJECT Currently there is no standardized tool for assessment of neurosurgical resident performance in the operating room. In light of enhanced requirements issued by the Accreditation Council for Graduate Medical Education's Milestone Project and the Matrix Curriculum Project from the Society of Neurological Surgeons, the implementation of such a tool seems essential for objective evaluation of resident competence. Beyond compliance with governing body guidelines, objective assessment tools may be useful to direct early intervention for trainees performing below the level of their peers so that they may be given more hands-on teaching, while strong residents can be encouraged by faculty members to progress to conducting operations more independently with passive supervision. The aims of this study were to implement a validated assessment tool for evaluation of operative skills in pediatric neurosurgery and determine its feasibility and reliability.

METHODS All neurosurgery residents completing their pediatric rotation over a 6-month period from January 1, 2014, to June 30, 2014, at the authors' institution were enrolled in this study. For each procedure, residents were evaluated by means of a form, with one copy being completed by the resident and a separate copy being completed by the attending surgeon. The evaluation form was based on the validated Objective Structured Assessment of Technical Skills for Surgery (OSATS) and used a 5-point Likert-type scale with 7 categories: respect for tissue; time and motion; instrument handling; knowledge of instruments; flow of operation; use of assistants; and knowledge of specific procedure. Data were then stratified by faculty versus resident (self-) assessment; postgraduate year level; and difficulty of procedure. Descriptive statistics (means and SDs) were calculated, and the results were compared using the Wilcoxon signed-rank test and Student t-test. A p value $<0.05$ was considered statistically significant.

RESULTS Six faculty members, 1 fellow, and 8 residents completed evaluations for 299 procedures, including 32 ventriculoperitoneal (VP) shunt revisions, 23 VP shunt placements, 19 endoscopic third ventriculostomies, and 18 craniotomies for tumor resection. There was no significant difference between faculty and resident self-assessment scores overall or in any of the 7 domains scores for each of the involved residents. On self-assessment, senior residents scored themselves significantly higher $(p<0.02)$ than junior residents overall and in all domains except for "time and motion." Faculty members scored senior residents significantly higher than junior residents only for the "knowledge of instruments" domain $(p=0.05)$. When procedure difficulty was considered, senior residents' scores from faculty members were significantly higher $(p=0.04)$ than the scores given to junior residents for expert procedures only. Senior residents' self-evaluation scores were significantly higher than those of junior residents for both expert $(p=0.03)$ and novice $(p=$ $0.006)$ procedures.

CONCLUSIONS OSATS is a feasible and reliable assessment tool for the comprehensive evaluation of neurosurgery resident performance in the operating room. The authors plan to use this tool to assess resident operative skill development and to improve direct resident feedback.

http://thejns.org/doi/abs/10.3171/2015.1.PEDS14511

KEY WORDS residency education; neurosurgery; surgery; operative skills; education assessment 
A LTHOUGH resident operative development is a key component of neurosurgical training, means of evaluating resident progress and skill acquisition are limited. Methods of evaluation of resident performance in the operating room, how skills develop over the course of training, and how to best quantify operative skills remain highly variable. ${ }^{8}$ While competency-based resident education has been previously suggested as a means of improving neurosurgical resident training, the optimal means of evaluating competency have not been established. ${ }^{11}$ Beginning in July 2013, the Neurological Surgery Milestone Project was launched as a joint initiative between the Accreditation Council for Graduate Medical Education (ACGME) and the American Board of Neurological Surgery (ABNS). These milestones are intended to be metrics by which residency programs can evaluate resident performance on a semiannual basis and report progress to the ACGME. Milestones include knowledge, attitude, and, notably, technical skills for each of the ACGME competencies. Residents are assigned a level of proficiency based on performance within each area of evaluation, independent of postgraduate year. Milestone performance data for each program will be evaluated by the Residency Review Committee as a component in the Next Accreditation System (NAS) to determine whether residents are progressing within a given program. ${ }^{19}$

These milestones provide a standardized method of evaluating performance, but they do not provide a standard approach to evaluation of operative skill. ${ }^{19}$ Even beyond implementation of these milestones, technical assessment tools have been difficult to implement and, therefore, have not become routine practice in most training programs. ${ }^{22}$ A user-friendly evaluation tool could greatly improve resident evaluation and feedback. After reviewing the general technical assessment tools available in the literature, ${ }^{1}$ we chose the Objective Structured Assessment of Technical Skills for Surgery (OSATS) global rating scale ${ }^{17}$ (Table 1). This tool, developed and implemented in the general surgery residency program at the University of Toronto, was selected because it is widely used and published in general surgery and has been validated for use in the clinical setting. 4,13,15,17 This rating scale has an advantage over other means of evaluation, as it is not limited to a specific procedure, as checklist-based evaluations often are. This permits a more comprehensive assessment of technical proficiency. ${ }^{17}$

Our aims were to implement a tool that assesses neurosurgical residents' technical performance in the operating room and to determine the feasibility and reliability of such a tool as it relates to self-assessment, postgraduate year (PGY) level, and procedure difficulty. Ideally, this tool could provide a standardized means of evaluating resident proficiency, as well as provide information about the level of operative skill that could be expected within an individual rotation and within the residency program as a whole.

\section{Methods}

\section{Cases and Research Participants}

Residents completing their pediatric neurosurgery rota- tions were evaluated using the OSATS tool. This included 3 PGY-3s, 1 PGY-4, and 4 PGY-6s. Evaluations were also completed for a pediatric neurosurgery fellow. These evaluations were considered for purposes of completion data but were not analyzed against the resident evaluations (i.e., the fellow was not considered a resident). Evaluation forms were made available for all pediatric neurosurgical procedures performed at Texas Children's Hospital between January 1, 2014, and June 30, 2014. Evaluations of resident performance during the case were completed by one of 6 attending-level neurosurgeons. The faculty members who operated with the residents performed the evaluation immediately after completion of the cases. Residents also completed the OSATS form to evaluate their own operative performance. To minimize recall bias, all evaluation forms were required to be completed before leaving the operating room. Faculty and self-evaluation scores for each resident were compared, overall and within each domain. The forms were filled out independently. Evaluations were compared overall and then considered by procedure, excluding those procedures for which there was only a self-evaluation or only a faculty evaluation. The rate of response for all surgical cases in the study time period was $85.1 \%$ for faculty members and $87 \%$ for residents. Participation in this study was optional.

The Baylor College of Medicine Institutional Review Board granted educational exemption status for this project.

\section{Assessment Tool}

Our evaluation form (Table 1) is based on the OSATS global rating scale. ${ }^{13,17}$ The validated Global Rating Scale of Operative Performance includes a 5-point Likert-type scale with 7 categories: respect for tissue; time and motion; instrument handling; knowledge of instruments; flow of operation; use of assistants; and knowledge of specific procedure. Reznick and others ${ }^{6-8,16,17,23}$ have validated use of this grading scale for performance evaluation in bench models of surgical tissues. This scale and its variations have also been used to evaluate general surgery resident performance in the operating room in studies similar to the present one., ${ }^{4,15}$

The faculty evaluators discussed use of the evaluation tool to achieve uniformity in operative performance interpretation and evaluation.

\section{Statistical Analysis}

We evaluated construct validity by examining the difference in performance scores between faculty assessment and self-assessment, both overall and in 7 domains of performance. Scores were also considered by resident level, with PGY-3s and PGY-4s considered together as junior residents and PGY-6s considered senior residents. Additionally, evaluations were compared by difficulty of procedure, designated either as novice or expert. These designations were assigned by a single faculty surgeon evaluator. Analysis was performed using commercially available Microsoft Excel and Stata 13 (StataCorp) statistical software. A p value $<0.05$ was considered significant. 
TABLE 1. Form used for evaluation of residents' operative skills

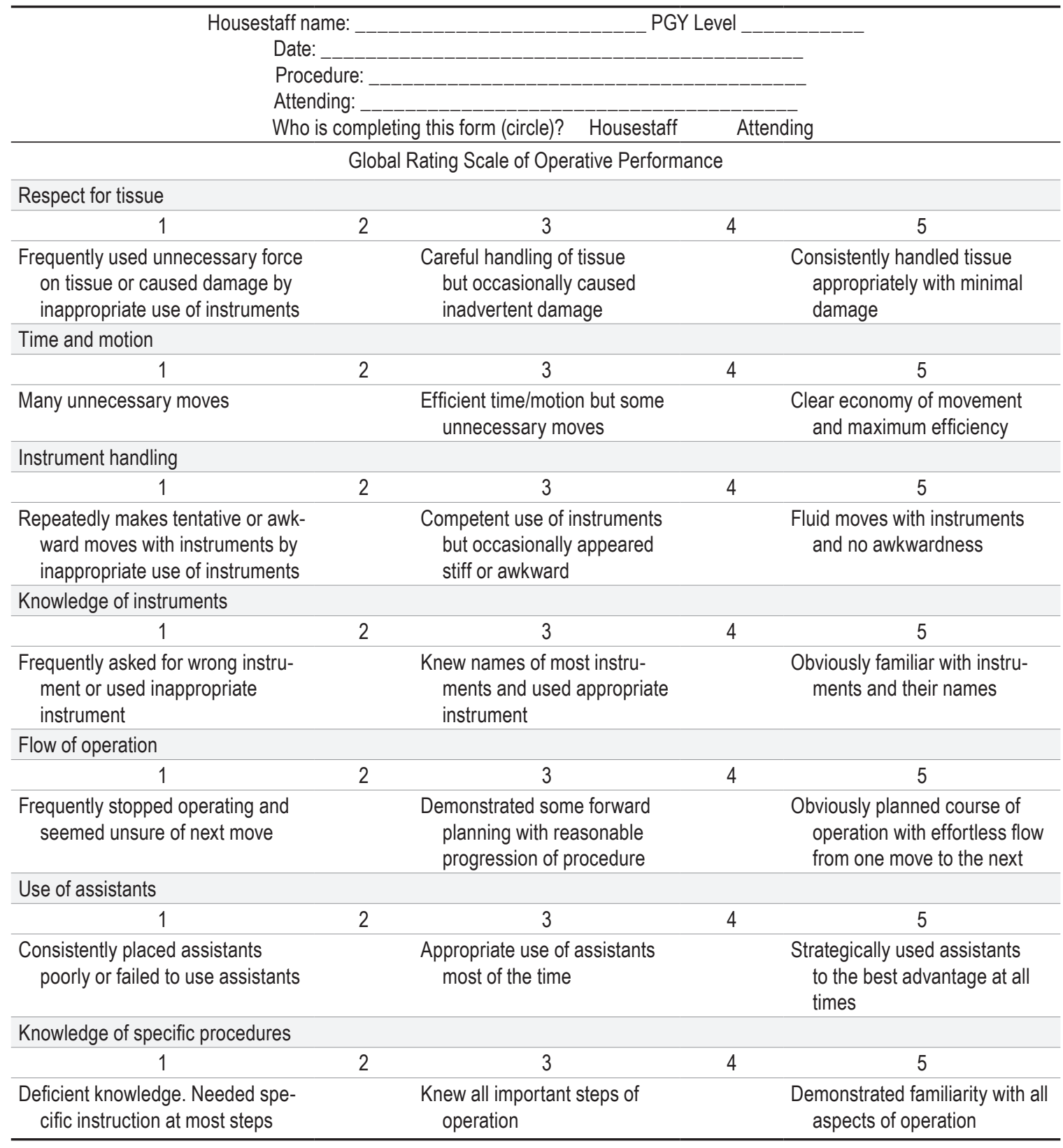

\section{Results}

\section{Feasibility}

Over a period of 6 months, 574 of 667 distributed assessments were completed, for a completion rate of $86 \%$. Evaluations reflected 299 procedures, which were categorized as novice or expert (Table 2). Each of the 6 facultylevel surgeons completed evaluations for $62 \%-98 \%$ of the operations they performed during the period of the study; 112 evaluations were completed for the junior residents, and 93 evaluations were completed for the senior residents. Evaluations completed for a fellow in pediatric neurosurgery were not considered for purposes of this study. Each of the 8 neurosurgery residents who rotated through pedi- atric neurosurgery during this 6-month period completed evaluations for $73 \%-100 \%$ of the surgical procedures in which they participated during the evaluation period. The high rate of completion suggests that the use of this assessment tool is feasible for long-term evaluation of resident performance. All evaluations were completed before leaving the operating room at the end of the case. No questions were left blank.

\section{Validity}

Resident and faculty evaluations were compared overall using the Wilcoxon signed-rank test (Table 3). A significant difference between resident and faculty ratings was 
TABLE 2. Novice- and expert-level procedures included in this study

\begin{tabular}{|c|c|}
\hline Novice & Expert \\
\hline Baclofen pump placement & AVM resection \\
\hline Baclofen pump removal & Anterior cervical fusion with corpectomy \\
\hline Bur hole drainage_abscess & Cervical and thoracic laminectomy and intradural tumor resection \\
\hline Bur hole drainage-subdural hematoma & Cervical decompression with posterior instrumented fusion \\
\hline C-1 laminectomy & Cranial remodeling for craniosynostosis \\
\hline Chiari decompression & Cranioplasty, LeFort III advancement \\
\hline Craniotomy for hematoma evacuation & Craniotomy for craniosynostosis \\
\hline Dermoid cyst resection & Craniotomy for grid placement \\
\hline Detethering of tethered cord & Craniotomy for resection of epileptic focus \\
\hline Durotomy closure & Craniotomy for tumor resection \\
\hline EVD placement & Endoscopic biopsy of third ventricle with VP shunt placement \\
\hline Excision of skull lesion & Endoscopic exploration of interhemispheric arachnoid cyst \\
\hline ICP monitor placement & Endoscopic sagittal synostectomy \\
\hline Internal pulse generator battery exchange & Endoscopic third ventriculostomy \\
\hline LP shunt revision & GPi electrode placement for deep brain stimulation \\
\hline Lumbar decompression & Lumbar laminectomy with posterior fusion \\
\hline Lumbar wound washout & Metopic synostectomy \\
\hline Lumbosacral laminectomy and discectomy & Moyamoya \\
\hline McComb reservoir placement & Myelomeningocele closure \\
\hline Occipital decompression & Occipitocervical fusion \\
\hline Ommaya reservoir placement & Pineal tumor resection \\
\hline Removal of bullet in neck & Posterior fossa craniotomy for tumor resection \\
\hline Selective dural rhizotomy & Posterior fossa tumor biopsy \\
\hline Vagus nerve stimulator battery exchange & Resection of right mastoid mass \\
\hline VP shunt externalization & Thoracic posterior fusion \\
\hline VP shunt placement & Transsphenoidal resection of tumor \\
\hline \multirow[t]{2}{*}{ VP shunt revision } & Vagus nerve stimulator placement \\
\hline & Vagus nerve stimulator revision \\
\hline
\end{tabular}

$\mathrm{AVM}=$ arteriovenous malformation; $\mathrm{EVD}=$ external ventricular drain; $\mathrm{GPi}$ = globus pallidus internus; $\mathrm{ICP}=$ intracranial pressure; $\mathrm{LP}=$ lumboperitoneal.

identified for overall scores $(\mathrm{p}=0.036)$ and in the domain "knowledge of procedures" $(p=0.043)$. No other significant differences were identified. However, when faculty evaluations and self-evaluations were sorted by resident year and compared, the Wilcoxon signed-rank test showed no significant difference between faculty evaluation and resident self-evaluation scores overall or in any of the 7 domains of evaluation for either junior or senior residents. The correlation between resident and faculty scores for a given resident in a given procedure was evaluated by calculating the Pearson's $r$ coefficient, which was 0.756 , indicating a strong correlation between resident and faculty ratings.

When faculty evaluations were compared between resident training levels, faculty members were found to score senior residents significantly higher than they scored junior residents in only 1 domain: "knowledge of instruments" ( $p=0.049)$. Otherwise, there was no significant difference in faculty scores for junior and senior residents. Self-evaluations showed a much greater difference between junior and senior residents. Senior residents scored themselves significantly higher than junior residents scored themselves both overall $(\mathrm{p}=0.007)$ and in all individual domains $(\mathrm{p}<0.02)$, except for "time and motion" (Table 4).

Evaluations were also considered based on the difficulty of the procedure being performed. In expert procedures, faculty members scored senior residents' performance significantly higher than that of junior residents $(\mathrm{p}=0.04)$; however, there was no significant difference in scores given to junior and senior residents by faculty members for novice procedures. Senior residents scored their own overall performance significantly higher than junior residents scored theirs for both expert $(\mathrm{p}=0.006)$ and novice $(\mathrm{p}=0.03)$ procedures (Table 5).

When faculty evaluations and self-evaluations from individual procedures were directly compared, all 4 junior residents gave themselves scores that differed significantly from those given by faculty surgeons. Three junior residents gave themselves lower scores overall than faculty members gave them $(\mathrm{p}<0.008)$, while 1 scored himself higher on self-evaluation than the faculty members scored 
TABLE 3. Overall comparison between resident and faculty evaluations*

\begin{tabular}{lccc}
\hline \multicolumn{1}{c}{ Domain } & $\begin{array}{c}\text { Attending } \\
\text { Evaluation }\end{array}$ & Self-Evaluation & p Value \\
\hline Overall & $4.04(0.73)$ & $3.75(0.72)$ & 0.036 \\
\hline Respect for tissue & $4.15(0.61)$ & $3.91(0.80)$ & 0.091 \\
\hline Time and motion & $3.89(0.76)$ & $3.60(0.76)$ & 0.161 \\
\hline Instrument handling & $4.06(0.85)$ & $3.69(0.80)$ & 0.068 \\
\hline Knowledge of instruments & $4.35(0.78)$ & $3.97(0.82)$ & 0.076 \\
\hline Flow of operation & $3.87(0.75)$ & $3.65(0.61)$ & 0.058 \\
\hline Use of assistants & $3.96(0.72)$ & $3.75(0.78)$ & 0.141 \\
\hline $\begin{array}{l}\text { Knowledge of specific } \\
\text { procedure }\end{array}$ & $3.97(0.69)$ & $3.68(0.69)$ & 0.043 \\
\hline
\end{tabular}

* Includes residents at all levels. Values are mean (SD) unless otherwise indicated. Bold type indicates statistical significance; $p$ values are based on the Wilcoxon signed-rank test.

him $(\mathrm{p}=0.04)$. In contrast, only 1 senior resident's selfevaluation differed significantly $(p<0.0007)$ from the scores given by the faculty surgeons.

\section{Discussion}

\section{Rationale for Establishing a Rating Tool}

One of the challenges of teaching clinical medicine and surgery is recognizing how much a trainee should be allowed to undertake on his own without allowing the patient to be harmed under the hands of an inexperienced practitioner. These decisions are largely made based on subjective assessment of resident skill and judgment; however, there is an increased effort in graduate medical education to implement standardized metrics to better assess resident competence and the overall quality of resident education. ${ }^{11}$ While the emphasis on competence assessment as a part of resident evaluation is not new, objective measures of neurosurgical trainee competence across a variety of procedures and skills have yet to be established. ${ }^{6,11}$ In the absence of an established standard for resident performance, it can be difficult to know what degree of competence is appropriate for given skills at a certain level. Interest in the objective assessment of these skills is growing rapidly. ${ }^{6}$
In 2009, the ACGME began a campaign to reorganize resident education based on the reported performance outcomes of residency programs within 6 prescribed areas of clinical competence, originally released in $2002 .^{14,21} \mathrm{~A}$ 2004 survey of neurosurgery residency program directors indicated that the majority of directors felt that these core competencies were difficult to understand and many others felt that the core competencies were not beneficial as metrics for resident progress or program success. ${ }^{12}$ While the core competencies may not independently improve resident evaluation, these efforts also included steps toward the implementation of standardized assessment methods. The first operative rating scale to be approved for use as a component of a program's evaluation system according to the new guidelines was the operative performance rating system (OPRS) from the Southern Illinois University School of Medicine. ${ }^{10}$ This system of evaluation relies on procedure-specific evaluation of resident performance in selected sentinel general surgery procedures..$^{10}$ Based on this rating scale, the OSATS was developed to evaluate performance independent of procedure. ${ }^{15}$ This assessment tool has been validated for use to evaluate the performance of general surgery trainees, but it has not been used to evaluate trainees in other surgical fields., ${ }^{2,3,8}$ Our study represents the first effort to implement this rating scale for evaluation of neurosurgery resident performance in the operating room. This rating scale can be used to direct feedback following a case, track resident progress during the course of the rotation, and give the residency training program information about areas in which there may be gaps in education.

\section{Using the OSATS for Evaluation of Neurosurgery Residents}

The OSATS is a comprehensive tool, but integration into the operating room workflow is feasible, as evidenced by the high completion rate of $86 \%$ observed in our study. Additionally, all of our evaluation forms were completed before leaving the operating room, as compared with other evaluation tools, such as the Southern Illinois University OPRS, which faculty members took an average of 12 days to complete in one study. ${ }^{9}$ Assessments performed in such a delayed fashion suffer from recall bias, affecting their validity. Early completion of assessments is important to

TABLE 4. Comparison of mean scores of PGY-3 and -4 and PGY-6 for residents overall and for each domain of the OSATS*

\begin{tabular}{|c|c|c|c|c|c|c|}
\hline \multirow[b]{2}{*}{ Domain } & \multicolumn{3}{|c|}{ Attending Evaluation } & \multicolumn{3}{|c|}{ Self-Evaluation } \\
\hline & PGY-3 and 4 & PGY-6 & p Value & PGY-3 and 4 & PGY-6 & $p$ Value \\
\hline Overall & $3.58(0.76)$ & $4.49(0.34)$ & 0.072 & $3.17(0.45)$ & $4.3(0.34)$ & 0.007 \\
\hline Respect for tissue & $3.75(0.62)$ & $4.55(0.27)$ & 0.057 & $3.27(0.51)$ & $4.5(0.44)$ & 0.009 \\
\hline Time and motion & $3.49(0.83)$ & $4.27(0.49)$ & 0.154 & $3.16(0.43)$ & $4.0(0.80)$ & 0.103 \\
\hline Instrument handling & $3.54(0.84)$ & $4.57(0.53)$ & 0.082 & $3.11(0.49)$ & $4.3(0.60)$ & 0.024 \\
\hline Knowledge of instruments & $3.84(0.82)$ & $4.87(0.18)$ & 0.049 & $3.30(0.54)$ & $4.6(0.31)$ & 0.005 \\
\hline Flow of operation & $3.38(0.73)$ & $4.36(0.39)$ & 0.054 & $3.16(0.40)$ & $4.1(0.26)$ & 0.006 \\
\hline Use of assistants & $3.49(0.72)$ & $4.42(0.36)$ & 0.060 & $3.10(0.47)$ & $4.4(0.29)$ & 0.003 \\
\hline Knowledge of specific procedure & $3.58(0.80)$ & $4.35(0.29)$ & 0.119 & $3.12(0.41)$ & $4.3(0.31)$ & 0.004 \\
\hline
\end{tabular}

* Values represent mean (SD) unless otherwise indicated; $p$ values are based on Student t-test. 
TABLE 5. Mean overall scores for PGY-3 and -4 and PGY-6 residents based on procedure difficulty in both faculty evaluations and self-evaluations*

\begin{tabular}{lcccccccc}
\hline \multirow{2}{*}{ Measure } & \multicolumn{3}{c}{ Expert-Level Procedures } & & & \multicolumn{3}{c}{ Novice-Level Procedures } \\
\cline { 2 - 3 } & PGY-6 & PGY-3 and 4 & p Value & & PGY-6 & PGY-3 and 4 & p Value \\
\hline Attending evaluation, overall average & $4.48(0.28)$ & $3.30(0.84)$ & $\mathbf{0 . 0 4 2}$ & & $4.48(0.46)$ & $3.64(0.76)$ & 0.112 \\
\hline Self-evaluation, overall average & $4.22(0.33)$ & $3.20(0.54)$ & $\mathbf{0 . 0 2 6}$ & & $4.6(0.27)$ & $3.22(0.46)$ & $\mathbf{0 . 0 0 6}$ \\
\hline
\end{tabular}

* Values represent mean (SD) unless otherwise indicated; $p$ values are based on Student t-test. Bold type indicates statistical significance.

provide more accurate results and more timely feedback for residents. Completion of the modified OSATS evaluation form is not time consuming, which may improve compliance and accuracy. Participants in our study were not asked to time their completion of the survey; however, previous studies using the same tools have found that completion takes between 2 and 5 minutes. ${ }^{4}$ Other explanations may be that the 6 faculty members involved in our study have a high interest in surgical education and were motivated to participate in the study. The high completion rate suggests that residents and faculty found the evaluation tool useful, as both groups appear to have prioritized survey completion even though completion was entirely voluntary.

Construct validity for neurosurgical residents was evaluated by comparison of evaluation scores between junior and senior residents and for novice and expert procedures. While faculty scores were significantly higher than resident scores both overall and in one of the domains of evaluation, this difference disappeared once scores were sorted by level of training. There were no significant differences between self-evaluation and faculty evaluation scores for either junior or senior residents in all domains of the scale, suggesting that the rating scale is, on the whole, a reliable representation of performance. This was further confirmed by the strong correlation between resident and attending scores $(r=0.756)$. When procedures were considered based on difficulty, average scores assigned by faculty surgeons for expert procedures were significantly higher for senior residents (Table 5), suggesting that this tool does reflect resident progress, particularly in performance of more complicated operative tasks.

When self-evaluation scores were compared between junior and senior residents, there was a significant difference between junior and senior resident scores overall and for all but 1 domain. However, when faculty surgeon scores for junior and senior residents were compared, there was a significant difference in only 1 domain of performance. This may indicate that residents' evaluations of their own skills are more sensitive to minor improvements, whereas faculty surgeons may be less sensitive to improvement in the performance of more simple procedures. The observation that there is a significant difference between faculty evaluation of junior and senior residents during more difficult procedures further supports this idea. This finding suggests that self-evaluation may be an important component of evaluation of resident progress.

While, overall, self-evaluation and faculty scores were not significantly different, when self-evaluation and faculty scores were compared on an individual resident basis, 3 of 4 junior residents scored themselves significantly lower than the faculty members scored them, while senior resident scores were individually more consistent with faculty evaluation. While this may simply represent a desire to not overestimate one's skill as a junior resident, it may indicate that residents become better able to assess the quality of their own performance as they progress through the training program.

\section{Limitations and Future Directions}

A major limitation of our study is that it was performed at a single institution. It is possible that the use of this assessment tool would produce different results at another institution. A second limitation is the small number of residents who rotate through the pediatric neurosurgery service and the number of faculty members on the pediatric neurosurgery staff. This study was also limited to a short time frame of only 6 months. Going forward, we plan to examine a longer period, ideally including multiple rotations and progression of resident level of training in the analysis. These preliminary findings are beneficial, however, as they may encourage others to adopt this assessment tool, permitting consideration of multiple institutions in future analyses and thus increasing the power of the results.

The small number of residents also limits the power of the current study. A sample calculation was performed for the OSATS using a predetermined power of $0.8, \mathrm{p}<0.05$, and a large effect size (Cohen's $d=0.86$ ). This indicated that 26 residents would be required to achieve these results. Our current sample size of 8 residents limits our ability to identify significant differences in performance and correlations between experience and performance. These 8 residents represent 8 out of a total of 12 residents who rotate in pediatric neurosurgery at our institution in a given year. This small class size calls for a multiinstitutional approach in future work to ensure a sufficient sample size.

An additional limitation is the unblinded nature of this study. Since the evaluators were the surgeons who operated with the resident, they were aware of the resident's identity, which may affect the results. Future directions for research may lead us to video-based assessment of resident operative skill, which can limit personal bias; however, this would also limit the domains that could be evaluated.

Our current study was also limited by the variability in the number of evaluation forms completed for each resident. The completed forms do not represent performance over the course of the entire pediatric neurosurgery rotation. Residents are expected to improve their performance on surgical procedures over a subspecialty-focused rotation, and evaluation of this progression of skill would be useful. ${ }^{8}$ In the future, we plan to evaluate the longitudinal 
validity of the OSATS evaluation tool during the pediatric neurosurgery rotation.

Additionally, our validation of the OSATS as a reproducible measure of competence was limited by lack of data to determine inter-attending variability in rating. Only one attending surgeon observed each procedure. There was considerable variation in the procedures and level of difficulty, and there was considerable variation in the number of evaluations each faculty surgeon completed for each resident. Because of this variation, we were unable to compare the scores given by multiple attending physicians to a given resident performing a single procedure to confirm reproducibility. Going forward, asking several faculty surgeons to evaluate the same resident during the same procedure could further validate the rating tool by permitting direct comparison between attending ratings. This comparison would then provide information about how reproducible the ratings are, independent of the attending surgeon. In the future, establishing a small number of common procedures for which multiple attending and self-evaluations could be compared would also allow for assessment of the internal validity of this tool.

In addition to fulfilling the requirements of the ACGME and ABNS, we plan to use this tool to provide meaningful feedback to residents. While in residency, trainees are expected to reach performance goals at each stage of training. Unfortunately, these goals are often not well defined nor are they always associated with detailed feedback to aid in meeting these achievement milestones. ${ }^{8,18,20}$ Providing timely and structured feedback to neurosurgery residents may help accelerate their learning curve. ${ }^{5,8}$ For this reason, we also aim to use this tool to provide residents with a better understanding of the level of their surgical skills as compared with the expectations for their stage of training, as well as helpful information to guide further improvement. Going forward, we plan to assess the utility of this tool in this capacity by asking residents to provide feedback about whether the OSATS was used to direct feedback from faculty and if they found that it improved their ability to implement suggestions for improvement.

\section{Conclusions}

The OSATS has been previously validated for evaluation of general surgery resident operative performance. ${ }^{15}$ We used this scale for evaluation of neurosurgery resident performance during the pediatric neurosurgery rotation, with the goal of validating this tool for use as a standard metric for assessment of resident performance. While there was not a significant difference across all domains between junior and senior resident performance, there was a significant difference between self-evaluation for junior and senior residents and a significant difference between faculty evaluation of junior and senior residents for procedures with an expert level of difficulty. These results represent preliminary evaluation of the OSATS for use in the evaluation of neurosurgery residents. While further work is warranted to confirm the reliability of this tool as a standardized assessment of resident skill, our findings suggest that the OSATS may be used to direct individual feedback to residents and advise on improvement of operative skills.
Furthermore, this tool may additionally be useful to document resident improvement, providing residency programs with meaningful information about resident competence and progress.

\section{References}

1. Ahmed K, Miskovic D, Darzi A, Athanasiou T, Hanna GB: Observational tools for assessment of procedural skills: a systematic review. Am J Surg 202:469-480, 480.e1-480.e6, 2011

2. Chipman JG, Schmitz CC: Using objective structured assessment of technical skills to evaluate a basic skills simulation curriculum for first-year surgical residents. J Am Coll Surg 209:364-370, 379.e1-370.e2, 2009

3. Choy I, Fecso A, Kwong J, Jackson T, Okrainec A: Remote evaluation of laparoscopic performance using the global operative assessment of laparoscopic skills. Surg Endosc 27:378-383, 2013

4. Doyle JD, Webber EM, Sidhu RS: A universal global rating scale for the evaluation of technical skills in the operating room. Am J Surg 193:551-555, 2007

5. Ericsson KA: Deliberate practice and acquisition of expert performance: a general overview. Acad Emerg Med 15:988994, 2008

6. Evans AW: Assessing competence in surgical dentistry. $\mathbf{B r}$ Dent J 190:343-346, 2001

7. Faulkner H, Regehr G, Martin J, Reznick R: Validation of an objective structured assessment of technical skill for surgical residents. Acad Med 71:1363-1365, 1996

8. Glarner CE, McDonald RJ, Smith AB, Leverson GE, Peyre S, Pugh CM, et al: Utilizing a novel tool for the comprehensive assessment of resident operative performance. J Surg Educ 70:813-820, 2013

9. Kim MJ, Williams RG, Boehler ML, Ketchum JK, Dunnington GL: Refining the evaluation of operating room performance. J Surg Educ 66:352-356, 2009

10. Larson JL, Williams RG, Ketchum J, Boehler ML, Dunnington GL: Feasibility, reliability and validity of an operative performance rating system for evaluating surgery residents. Surgery 138:640-649, 2005

11. Long DM: Competency-based residency training: the next advance in graduate medical education. Acad Med 75:11781183,2000

12. Lunsford LD, Kassam A, Chang YF: Survey of United States neurosurgical residency program directors. Neurosurgery 54:239-247, 2004

13. Martin JA, Regehr G, Reznick R, MacRae H, Murnaghan J, Hutchison C, et al: Objective structured assessment of technical skill (OSATS) for surgical residents. Br J Surg 84:273278, 1997

14. Nasca TJ, Philibert I, Brigham T, Flynn TC: The next GME accreditation system-rationale and benefits. $\mathbf{N}$ Engl J Med 366:1051-1056, 2012

15. Niitsu H, Hirabayashi N, Yoshimitsu M, Mimura T, Taomoto J, Sugiyama Y, et al: Using the Objective Structured Assessment of Technical Skills (OSATS) global rating scale to evaluate the skills of surgical trainees in the operating room. Surg Today 43:271-275, 2013

16. Regehr G, MacRae H, Reznick RK, Szalay D: Comparing the psychometric properties of checklists and global rating scales for assessing performance on an OSCE-format examination. Acad Med 73:993-997, 1998

17. Reznick R, Regehr G, MacRae H, Martin J, McCulloch W: Testing technical skill via an innovative "bench station" examination. Am J Surg 173:226-230, 1997

18. Rose JS, Waibel BH, Schenarts PJ: Disparity between resident and faculty surgeons' perceptions of preoperative prepa- 
ration, intraoperative teaching, and postoperative feedback. J Surg Educ 68:459-464, 2011

19. Selden N (ed): The Neurological Surgery Milestone Project. Chicago: Accreditation Council for Graduate Medical Education; Woodbridge, CT: American Board of Neurological Surgery, 2013. (http://www.acgme.org/acgmeweb/Portals/0/PDFs/Milestones/NeurologicalSurgeryMilestones.pdf) [Accessed June 29, 2015]

20. Snyder RA, Tarpley MJ, Tarpley JL, Davidson M, Brophy C, Dattilo JB: Teaching in the operating room: results of a national survey. J Surg Educ 69:643-649, 2012

21. Swing SR, Clyman SG, Holmboe ES, Williams RG: Advancing resident assessment in graduate medical education. $\mathbf{J}$ Grad Med Educ 1:278-286, 2009

22. van Hove PD, Tuijthof GJ, Verdaasdonk EG, Stassen LP, Dankelman J: Objective assessment of technical surgical skills. Br J Surg 97:972-987, 2010

23. Winckel CP, Reznick RK, Cohen R, Taylor B: Reliability and construct validity of a structured technical skills assessment form. Am J Surg 167:423-427, 1994

\section{Disclosure}

The authors report no conflict of interest concerning the materials or methods used in this study or the findings specified in this paper.

\section{Author Contributions}

Conception and design: Jea, Lam. Acquisition of data: Jea, Briceño. Analysis and interpretation of data: Jea, Hadley, Lam, Briceño. Drafting the article: Jea, Hadley, Lam. Critically revising the article: Jea, Lam, Briceño, Luerssen. Reviewed submitted version of manuscript: all authors. Approved the final version of the manuscript on behalf of all authors: Jea. Administrative/technical/ material support: Luerssen. Study supervision: Lam, Luerssen.

\section{Correspondence}

Andrew Jea, Division of Pediatric Neurosurgery, Department of Neurosurgery, Texas Children's Hospital, Baylor College of Medicine, 6621 Fannin St., CCC 1230.01, 12th Fl., Houston, TX 77030.email: ahjea@texaschildrens.org. 